\section{OPEN ACCESS}

Edited by:

Paulus Stefan Rommer,

Medical University of Vienna, Austria

Reviewed by:

Ingo Kleiter,

Ruhr University Bochum, Germany

John Corboy,

University of Colorado, United States

Timothy Vollmer,

University of Colorado Anschutz

Medical Campus, United States

*Correspondence:

Tim Spelman

tim.spelman@ki.se

Specialty section:

This article was submitted to

Multiple Sclerosis and

Neuroimmunology,

a section of the journal

Frontiers in Neurology

Received: 30 December 2020

Accepted: 15 February 2021

Published: 17 March 2021

Citation:

Hillert J, Magyari M, Soelberg Sørensen P, Butzkueven H, Van Der

Welt A, Vukusic S, Trojano $M$,

laffaldano $P$, Pellegrini $F$, Hyde $R$,

Stawiarz L, Manouchehrinia A and

Spelman T (2021) Treatment

Switching and Discontinuation Over 20 Years in the Big Multiple Sclerosis

Data Network.

Front. Neurol. 12:647811

doi: 10.3389/fneur.2021.647811

\title{
Treatment Switching and
} Discontinuation Over 20 Years in the Big Multiple Sclerosis Data Network

\begin{abstract}
Jan Hillert ${ }^{1}$, Melinda Magyari ${ }^{2,3}$, Per Soelberg Sørensen ${ }^{2}$, Helmut Butzkueven ${ }^{4,5}$, Anneke Van Der Welt ${ }^{4}$, Sandra Vukusic ${ }^{6,7,8}$, Maria Trojano $^{9}$, Pietro laffaldano ${ }^{9}$, Fabio Pellegrini ${ }^{10}$, Robert Hyde ${ }^{10}$, Leszek Stawiarz ${ }^{1}$, Ali Manouchehrinia ${ }^{1}$ and Tim Spelman ${ }^{1,4 *}$ on behalf of the Big MS Data Network (BMSD): a collaboration of the Danish MS Registry, Italian MS Registry, the Observatoire Français de la Sclérose en Plaques (OFSEP) registry, the MSBase Study Group and the Swedish MS Registry
\end{abstract}

${ }^{1}$ Department of Clinical Neuroscience, Karolinska Institute, Stockholm, Sweden, ${ }^{2}$ The Danish Multiple Sclerosis Registry, Department of Neurology, Rigshospitalet, Copenhagen, Denmark, ${ }^{3}$ Department of Neurology, Danish Multiple Sclerosis Center, Rigshospitalet, University of Copenhagen, Copenhagen, Denmark, ${ }^{4}$ MSBase Foundation, Melbourne, VIC, Australia, ${ }^{5}$ Multiple Sclerosis and Neuroimmunology Research, Central Clinical School, Alfred and Box Hill Hospitals, Monash University, Melbourne, VIC, Australia, ${ }^{6}$ Service de Neurologie, sclérose en plaques, pathologies de la myéline et neuro-inflammation, and Observatoire Français de la Sclérose en Plaques, Hôpital Neurologique Pierre Wertheimer, Hospices Civils de Lyon, Lyon, France, ' Centre des Neurosciences de Lyon, INSERM 1028 et CNRS UMR5292, Lyon, France, ${ }^{8}$ Université Claude Bernard Lyon 1, Faculté de Médicine Lyon-Est, Lyon, France, ${ }^{9}$ Department of Basic Medical Sciences, Neurosciences, and Sense Organs, University of Bari Aldo Moro, Bari, Italy, ${ }^{10}$ Biogen International GmbH, Zug, Switzerland

Background: Although over a dozen disease modifying treatments (DMTs) are available for relapsing forms of multiple sclerosis (MS), treatment interruption, switching and discontinuation are common challenges. The objective of this study was to describe treatment interruption and discontinuation in the Big MS data network.

Methods: We merged information on 269,822 treatment episodes in 110,326 patients from 1997 to 2016 from five clinical registries in this cohort study. Treatment stop was defined as a clinician recorded DMT end for any reason and included treatment interruptions, switching to alternate DMTs and long-term or permanent discontinuations.

Results: The incidence of DMT stopping cross the full observation period was lowest in FTY (19.7 per 100 person-years (PY) of treatment; 95\% Cl 19.2-20.1), followed by NAT (22.6/100 PY; 95\% Cl 22.2-23.0), IFNß (23.3/100 PY; 95\% Cl 23.2-23.5). Of the 184,013 observed DMT stops, 159,309 (86.6\%) switched to an alternate DMT within 6 months. Reasons for stopping a drug were stable during the observation period with lack of efficacy being the most common reason followed by lack of tolerance and side effects. The proportion of patients continuing on most DMTs were similarly stable until 2014 and 2015 when drop from 83 to $75 \%$ was noted.

Conclusions: DMT stopping reasons and rates were mostly stable over time with a slight increase in recent years, with the availability of more DMTs. The overall results suggest that discontinuation of MS DMTs is mostly due to DMT properties and to a lesser extent to risk management and a competitive market.

Keywords: multiple sclerosis, disease modifying treatment, big MS data, registry study, treatment interruption and discontinuation 


\section{INTRODUCTION}

Multiple sclerosis (MS) is a life-long disease where disability typically develops over decades. For over 20 years, disease modifying treatments (DMTs) have been available to reduce attack frequencies, focal inflammatory brain lesions and development of disability. Although over a dozen DMTs are available for relapsing forms of multiple sclerosis (MS), discontinuation of treatment is a common challenge as consistent control of inflammation is a priority (1-5). Conversely, the broader availability of an increasingly diverse range of treatment options provides opportunities for better management in patients able to switch. Changes in product availability, reimbursement and treatment recommendations have led to a growing interest for identifying reliable predictors of DMT discontinuation (6-9). Furthermore, discontinuation on a prescribed drug may differ between specific drugs, between countries, over time and calendar year (of licensing new drug) (10-12).

The growth of real-world clinical MS databases, particularly large, longitudinal disease registries, provide a unique opportunity to describe discontinuation trends over large patient numbers and long-term observation periods $(13,14)$. The objective of this study was to describe the frequency of DMT discontinuation recorded across the pooled Big MS Data Network (BMSD) and to descriptively compare patterns of discontinuation between different time periods and treatment epochs.

\section{MATERIALS AND METHODS}

\section{Data}

The project was conducted using data from the five clinical MS registries included in the BMSD network project: the Italian MS registry, OFSEP of France, the Danish and Swedish national MS registries and the international MSBase database. Treatment episodes and associated patient data complying to minimum dataset requirements were individually extracted from the five contributing datasets and then pooled into a single combined dataset. Minimum dataset requirements included treatment information (product, treatment start and end dates and reason for treatment interruption/discontinuation), demography (age and sex) and clinical and disease characteristics (date of first symptoms, date of MS diagnosis, EDSS).

\section{Quality Checks}

Data quality checks were conducted prior to merging to minimize outcome assessment and follow-up bias in the cohorts under study. This included ensuring all variables required for the minimum analysis dataset had been extracted and transferred in the correct format, consistent across all five registries. The data was then checked for duplicates and date inconsistencies covering key demographic, disease and treatment dates. Data counts were then performed to assess completeness of key variables.

\section{Patients and Observation Period}

Diagnosis of MS was confirmed according to Poser or McDonald criteria. All eligible MS patients for whom at least one DMT had been initiated during the relapsing remitting MS stage of disease were included in the analysis. Subjects were considered exposed to a DMT if they had received at least one injection/infusion (or at least a one-time consumption of an oral drug). We defined a prestudy period preceding the index date during which patients were required to have continuous medical service coverage. This was defined as a minimum of 6 months. The pre-study period ensured a standard run-in period without DMT exposure and a standard period during which the diagnosis of MS was identified. Patients not diagnosed as RRMS or RRMS patients not initiating a DMT therapy during the RRMS phase were excluded from the analysis. The observation period of the study was January 1st 1996 through 31st December 2016. Patients were included in the analysis if they recorded at least one qualifying DMT episode at any stage during the observation period and were not required to be active registry follow-up for the entirety of the observation period. A subset of patients from the Italian registry are also tracked in MSBase. To avoid duplicate patient records these patients were removed from the MSBase contribution to the overall pooled sample.

\section{Definitions}

A treatment episode was defined as the time from clinician recorded DMT start to clinician recorded DMT stop. For convenience, we have referred here to all DMT interruptions, switching and discontinuations as treatment changes. Treatment stop was defined as a clinician recorded DMT end for any reason. This included both DMT stops that were followed by a switch or change to an alternate DMT and DMT stops that were followed by no treatment for the remainder of a patient's follow-up. A treatment switch was defined as a gap of no $>6$ months between ceasing a DMT and initiating an alternate DMT. Changes of dose of an existing DMT or the addition of a second drug were not considered to represent discontinuations. DMTs included in the analysis were categorized as followed: IFN $\beta$-1a IM, IFN $\beta$-1a SC, IFN $\beta$-1b, glatiramer acetate, IFN $\beta$-1a not further specified, natalizumab, rituximab, fingolimod, dimethyl fumarate, teriflunomide, alemtuzumab and other. Reasons for discontinuation were analyzed as reported by the contributing registries.

\section{Ethics Statement}

The datasets presented in this article are not readily available secondary to the governing rules of the contributing registries that prohibit the sharing of both patient level data and aggregate data that may identify individual patients. Selected data and analyses may be accessed via direct request to the individual registries and subject to satisfying the data sharing permission rules of each registry.

\section{Statistical Analyses}

This analysis was descriptive only. Categorical variables were summarized using frequency and percentage. Continuous variables were summarized using mean and standard deviation (SD) or median and inter-quartile range (IQR) as 
TABLE 1 | Treatment episodes and discontinuations by registry.

\begin{tabular}{|c|c|c|c|c|c|c|c|}
\hline & Category & Denmark & Sweden & OFSEP & Italy & MSBase & Total \\
\hline \multicolumn{8}{|l|}{ PATIENT CHARACTERISTICS ${ }^{*}$} \\
\hline Patient count $-\mathrm{n}$ & & 7990 & 15983 & 24616 & 26985 & 34752 & 110326 \\
\hline & Male & 2505 (31.4) & 4738 (29.6) & $6283(25.5)$ & 8670 (32.1) & $9861(28.4)$ & $32057(29.1)$ \\
\hline Age at MS onset (years) - mean (SD) & & $32.8(9.9)$ & $34.4(12.8)$ & $31.1(9.5)$ & $29.6(9.7)$ & $30.5(9.9)$ & 30.9 (10.3) \\
\hline Treatment episodes $-\mathrm{n}$ & & 14252 & 38229 & 65535 & 79816 & 71990 & 269822 \\
\hline Discontinuations - $\mathrm{n}(\%)$ & & $8936(62.7)$ & $24704(64.6)$ & $45966(70.1)$ & $59590(74.7)$ & $44817(62.3)$ & $184013(68.2)$ \\
\hline Treatment duration (years) - mean (SD) & & $2.82(2.38)$ & $2.31(2.24)$ & $2.18(2.18)$ & $2.06(2.12)$ & $2.29(2.20)$ & $2.23(2.20)$ \\
\hline
\end{tabular}

${ }^{*}$ Count of individual patients contributing at least 1 treatment episode to the analysis.
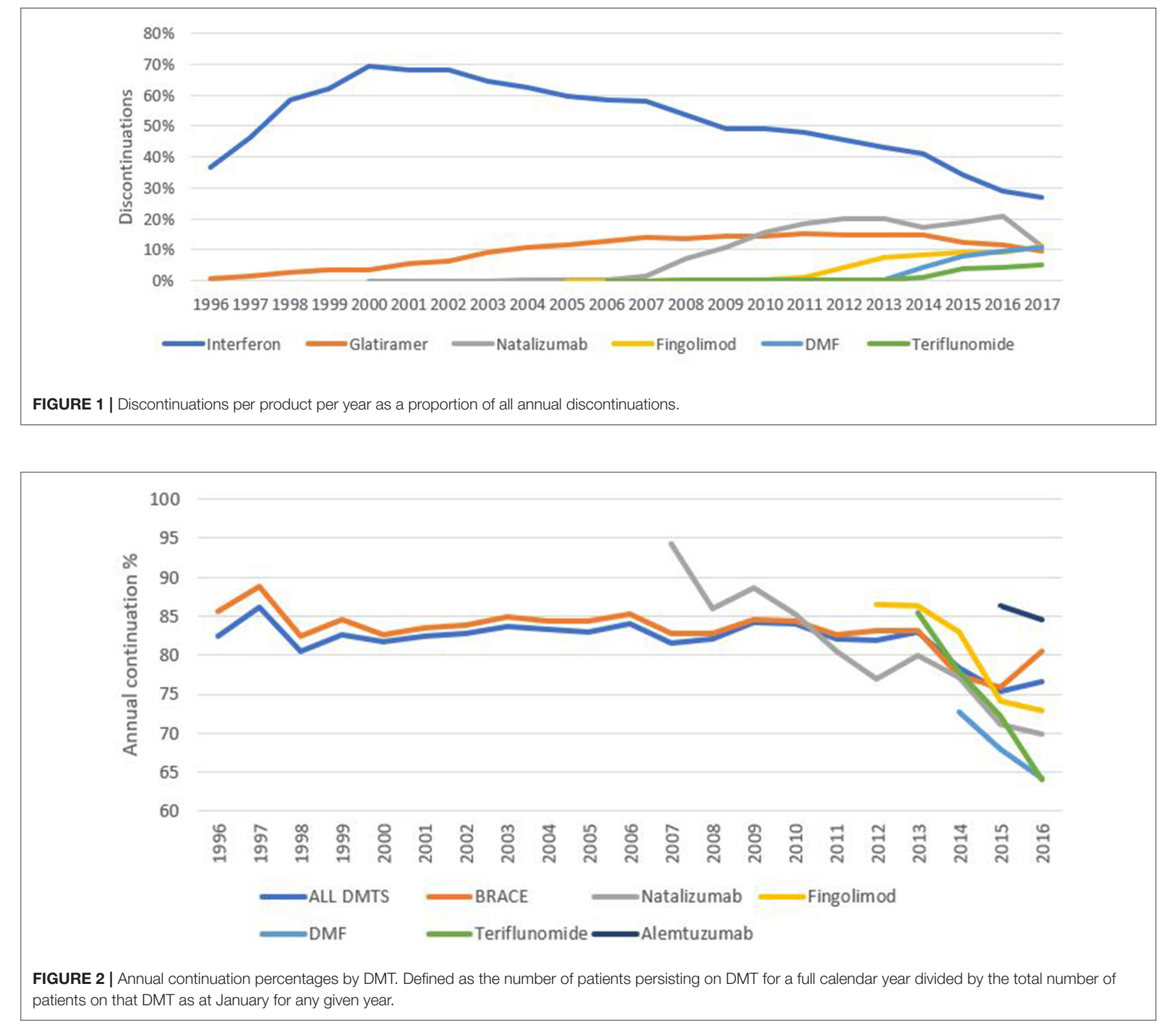
TABLE 2 | Reason for discontinuation.

\begin{tabular}{|c|c|c|}
\hline & Category & Total \\
\hline Discontinuations & & $n=184,013$ \\
\hline \multirow[t]{13}{*}{$\begin{array}{l}\text { Reason for } \\
\text { discontinuation }-n(\%)\end{array}$} & $\begin{array}{l}\text { Inadequate efficacy } \\
\text { Disease progression/EDSS } \\
\text { progression/EDSS } 7+\end{array}$ & $\begin{array}{c}26,034(14.1) \\
4,218(2.3)\end{array}$ \\
\hline & Tolerability & $15,491(8.4)$ \\
\hline & Adverse event/side effects/safety & $18,145(9.9)$ \\
\hline & Allergic reaction & $3,537(1.9)$ \\
\hline & Convenience & $8,428(4.6)$ \\
\hline & $\begin{array}{l}\text { Pregnancy (planned or confirmed), } \\
\text { contraception cessation }\end{array}$ & $6,733(3.7)$ \\
\hline & Scheduled stop & $12,147(6.6)$ \\
\hline & $\begin{array}{l}\text { Non-adherence/non-compliance/no } \\
\text { motivation }\end{array}$ & $4,555(2.5)$ \\
\hline & $\begin{array}{l}\text { Development of neutralizing } \\
\text { antibodies }\end{array}$ & $1,273(0.7)$ \\
\hline & Deceased & $266(0.1)$ \\
\hline & Secondary progressive MS & $1,109(0.6)$ \\
\hline & Other & $10,506(5.7)$ \\
\hline & Not reported/unknown & $76,887(41.8)$ \\
\hline
\end{tabular}

EDSS, Expanded disability status scale; MS, Multiple sclerosis.

appropriate. All analyses were conducted in $\mathrm{R}$ ( $\mathrm{R}$ Foundation for Statistical Computing).

\section{RESULTS}

A total of 110,326 patients contributing 269,822 DMT treatment episodes from the five registries were included in the analysis (Table 1). Across the pooled sample, females accounted for $78,269(70.9 \%)$ of included patients. This proportion was similar across cohorts ranging from a low of $67.9 \%$ in the Italian cohort to a high of $74.5 \%$ in the French OFSEP cohort. Mean (SD) age at MS onset was 30.9 years (10.3). Mean (SD) age at DMT initiation was 36.6 years (11.0) across the entire cohort. Across the observation period, Scandinavian patients tended to be treated later, with Danish patients initiating DMT at a mean (SD) of 38.3 (12.8) years of age and Swedish patients starting treatment at a mean (SD) of 40.7 (12.4) years. This compares to a mean of $35.5,35.8$ and 36.3 years, respectively, for the MSBase, Italian and French cohorts, respectively. Across the full observation period, mean (SD) treatment duration was comparable across all five registries, ranging from 2.06 (2.12) years in Italian patients up to 2.82 years $(2.38)$ in the Danish cohort.

A total of $184,013(68.2 \%)$ DMTs were stopped during the observation period. IFN $\beta$ accounted for the largest proportion of observed treatments in the pooled data $(116,551$ treatment episodes; 43.2\%), followed by natalizumab (NTZ) (33,974; $12.6 \%)$, glatiramer acetate (GLA) $(32,324 ; 12.0)$ and fingolimod (FTY) $(19,675 ; 7.3 \%)$. The incidence of DMT stopping across the full observation period was lowest in FTY (19.7 stops per 100 person-years (PY) of treatment; 95\% CI 19.2-20.1), followed by NAT (22.6/100 PY; 95\% CI 22.2-23.0), IFN及 (23.3/100 PY; 95\% CI 23.2-23.5) and GLA (25.8/100 PY; 95\% CI 25.4-26.2). Across the pooled data there was a wide variety of treatment pathways taken by patients following stopping of first line therapy. Of the 184,013 observed DMT stops, 159,309 (86.6\%) switched to an alternate DMT within 6 months of discontinuation. The most frequent switch products across the observation period were an alternate platform (IFN $\beta$ or glatiramer), natalizumab or fingolimod.

Consistent with being the most frequently prescribed DMT, IFN was the most frequently discontinued DMT in each of the years between 1996 and 2017 inclusive (Figure 1), accounting at its peak for $69.1 \%(2,230 / 3,228)$ of all DMT stops that occurred during the year 2000. IFN accounted for the majority of the market share at this time, accounting for $76.7 \%(4,743 / 6,181)$ of all patients treated with DMT at the beginning of 2000 . This gradually fell to a low of $26.8 \%$ of all treatment interruptions $(922 / 3,441)$ in 2017. Glatiramer acetate was the second most frequently discontinued DMT between 1996 and 2009 (accounting for 7,287/69,647 treatment interruptions during this period), before being overtaken by natalizumab in 2010, which contributed 19\% $(2,026 / 10,680)$ of DMT stops during that year. By 2017, four drugs (NTZ 11.1\%, FTY $11.1 \%$, Dimethyl Fumarate $10.8 \%$ and GLA 9.4\%) accounted for almost equal proportions of DMT stops, accounting for 319, 319, 310, and 270 of the total 2,870 treatment interruptions recorded in that year. Of all patients on DMT at the start of 2017, FTY, GLA, NTZ, and DMF accounted for 16.0, 12.3, 9.1, and $6.2 \%$ of these treatments, respectively.

\section{Persistence}

A key goal in MS management is to provide patients with a DMT they are likely to continue on over a longer period of time. Figure 2 illustrates the treatment-specific chance of continuing for another year on (a) all drugs combined as well as for (b) platforms (IFN $\beta$-1a IM, IFN $\beta$-1a SC, IFN $\beta$-1b, and glatiramer acetate) or (c) natalizumab. Interestingly, overall continuation is fairly stable until the last few years when a decrease was observed in 2014 and 2015 for all drugs combined as well as for the platform DMT group. In contrast, the annual chance of continuing on natalizumab has gradually decreased from $95 \%$ during its first year to $70 \%$ in 2016, likely as a consequence of a gradual implementation of a risk management scheme to prevent progressive multifocal leukoencephalopathy (PML).

\section{Reason for DMT Stopping}

Where reason for treatment stopping was documented, lack of efficacy was the most frequently reported across the pooled data $(23.2 \%)$, followed by adverse or side effects $(16.1 \%)$ and intolerance (13.8\%) across the full time period (Table 2). In terms of data quality, the proportion of treatment stops not reporting a reason for discontinuation had steadily declined across all registries from an average of $68 \%$ in 1996 down to just $32 \%$ in 2016 (Figure 3). There was however, some variability in this reduction by DMT/drug class with, for example, alemtuzumab associated with just $9 \%$ of discontinuations not reporting a reason in 2016, compared with $26 \%$ of the platform DMTs (Figure 4). 


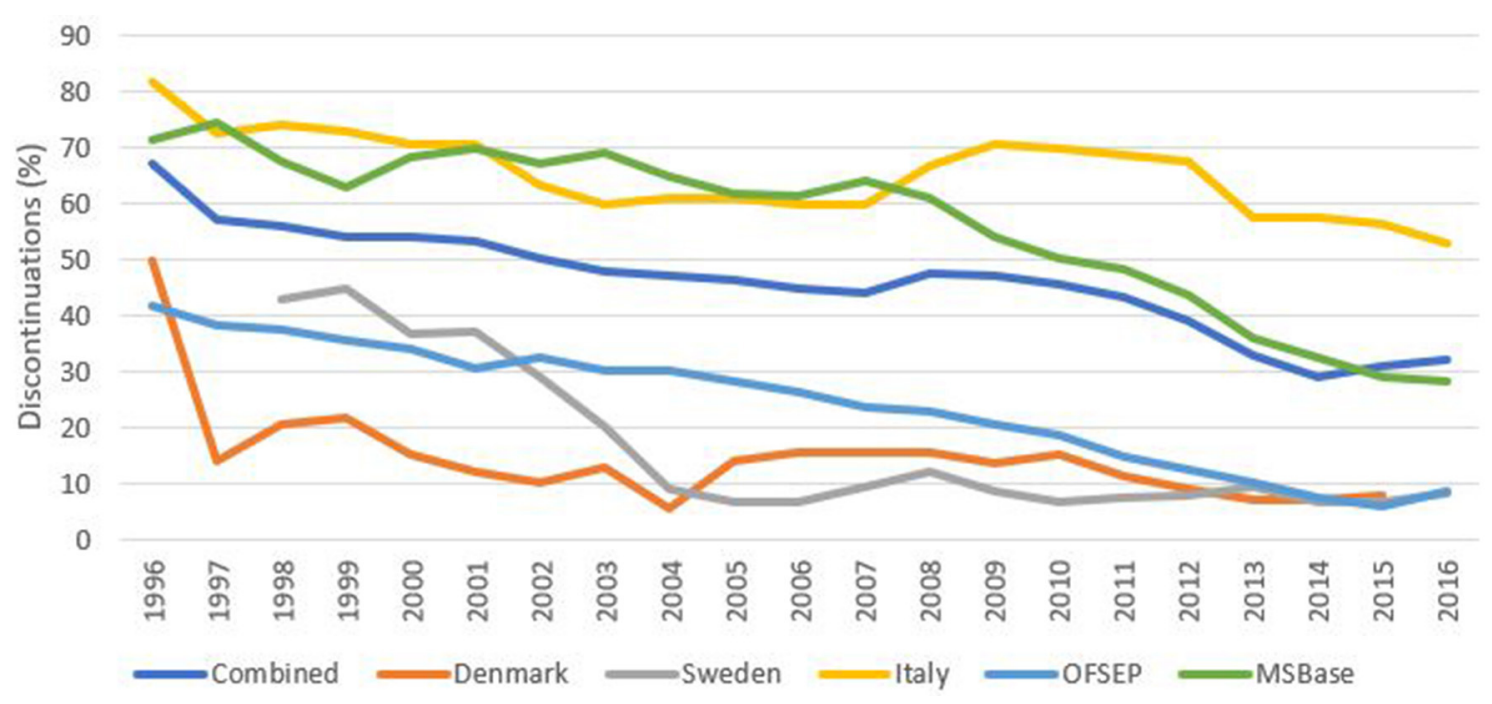

FIGURE 3 | Percentage of discontinuations not reporting a discontinuation reason by registry.

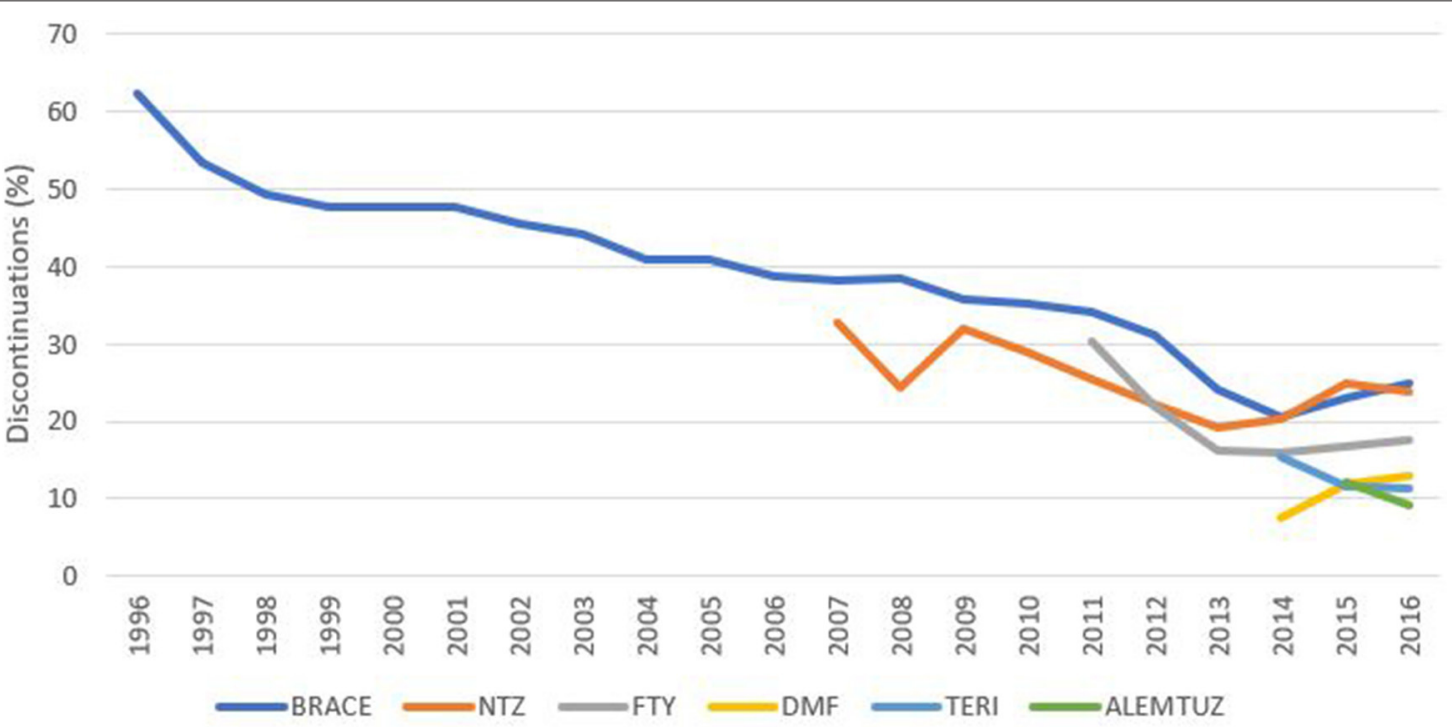

FIGURE 4 | Percentage of discontinuations not reporting a discontinuation reason by DMT.

\section{DISCUSSION}

Stopping disease-modifying treatment in MS is a common event, both across products and treatment epochs. Frequent treatment interruption, switching and discontinuation remains a major challenge for replicating the treatment efficacy observed in pivotal clinical trials in real-world clinical practice. In this descriptive analysis, we have compiled the largest ever dataset of MS-specific DMT treatment episodes to describe, quantify and illustrate patterns of discontinuation in an increasingly complex treatment environment. Thanks to standardization of variable definitions, and similarities in data structure between the leading MS registries contributing treatment data to the analysis, we were able to merge data files on almost 270,000 treatment episodes in just over 110,000 patients from five major MS registries. In this analysis, we focused the evolution of stopping, switching and discontinuation patterns over 20 years, from 1997 to 2016.

Perhaps the most striking feature of this descriptive analysis is large number of complex switching patterns and treatment pathways observed, with many patients moving from one DMT to another. The range and diversity of treatment pathways have multiplied over time as new treatments have entered the market. Disaggregating the analysis by the three most common treatment groups (IFN- $\beta$, glatiramer acetate and natalizumab), we observed greater complexity and diversity in treatment trajectories in patients initiating either first-line interferon-beta or glatiramer, 
relative to natalizumab. This is consistent with the longer time on the market of the two platform treatment groups. The early domination of the market by Interferon- $\beta$ ensures these products dominate the discontinuation data over the observation period (15). However, the expansion of approved products for MS has led to a much more diverse discontinuation picture in the more recent years (Figure 1).

Regarding reasons for discontinuation, the pattern has been remarkably stable over the study period with lack of efficacy being the most common reason followed by lack of tolerance, side effects, convenience and disease progression in that order (Table 2 and Figure 2). However, these are likely to become more complex and more heterogenous as novel treatments and agents are added to the battery of available DMTs for MS (16, 17). Likewise, the proportion of patients continuing on a drug they are already prescribed has over the years been stable up until 2014 when a clear change took place which continued the following year to bring down overall continuation chance from 83 to $75 \%$. This change may have been prompted by the introduction on the markets of novel per-oral drugs such as dimethyl fumarate and teriflunomide. Natalizumab, on the other hand, has shown a gradually decreased continuation rate each year after its introduction.

In spite of its size, this descriptive study has several limitations. Reason for discontinuation data was not reported for $41.8 \%$ of DMT stops. The amount of missing discontinuation reason data varied by registry, from a low of $10.5 \%$ of discontinuations in the Danish MS register, up to $63.5 \%$ in the Italian registry. Whilst this left a total of 107,126 DMT discontinuations with reasons reported to analyze, it remains a major limitation of this study, particularly as were limited to analyzing treatment stop reason to exactly how they were reported in each registry. However, discontinuation reason reporting greatly improved over the observation period and we would anticipate this trend would continue as the registries evolve further. Whilst overall there was good consistency in the type of discontinuation reason categories collected by each registry, there were some notable exceptions. Convenience was not available as a separate option in the Swedish registry and thus the convenience data is likely to represent an under-estimate of the reality. It also may explain why the "other reason" category was the most frequently reported discontinuation reason category in the Swedish data, being likely to include treatment switches made on the grounds of convenience. Similarly, ceasing treatment secondary to neutralizing antibodies was only present in the Danish and Swedish registries. There is also likely to be some overlap between related categories such as "lack of efficacy" and "disease progression," or "adverse events," "side effects," and "lack of tolerance." Furthermore, JCV status, an important determinate of pre-mature natalizumab discontinuation, was not sufficiently available to permit analysis or disaggregation. Finally, comparisons of proportions and percentages are strictly descriptive, with no statistical adjustment made for clinical or practice-based factors that may also influence persistence or discontinuation $(8,18,19)$. Formal statistical comparisons of clinical outcomes by drug class or treatment pattern and sequence form, adjusted for patient and disease factors, the basis of a parallel study currently being undertaken by the study group. Future directions also included the formal analysis of drivers of, and outcomes from, different treatment switch patterns.

While our results show some changes in DMT stopping over time, likely reflecting the availability of alternative treatments, typically increasing over time, changes are mainly modest and gradual, suggesting that the main driver of change of treatment is likely to be properties of the various drugs, their formulations and routes of administration.

\section{DATA AVAILABILITY STATEMENT}

The availability of the datasets presented in this article are limited by the governing rules of the contributing registries that prohibit the sharing of both patient level data and aggregate data that may identify individual patients. Selected data and analyses may be accessed via direct request to the individual registries and subject to satisfying the data sharing permission rules of each registry.

\section{ETHICS STATEMENT}

The studies involving human participants were reviewed and approved by each contributing registry to the Big MS Data Network according to their own ethics, operating and inclusion rules. Each registry is required to obtain their own approval prior to provision of the data and pooling.

\section{AUTHOR'S NOTE}

All authors/investigators from the Italian MS Registry, OFSEP and the MSBase Study Group are listed in the Supplementary Material.

\section{AUTHOR CONTRIBUTIONS}

TS was involved in the study design, data analysis, interpretation, manuscript preparation, and review. MM, PS, HB, AV, SV, MT, $\mathrm{PI}, \mathrm{FP}, \mathrm{RH}, \mathrm{LS}, \mathrm{AM}$, and $\mathrm{JH}$ was involved in the study design, interpretation, and manuscript review. All authors contributed to the article and approved the submitted version.

\section{FUNDING}

This study was supported by an unrestricted grant from Biogen.

\section{SUPPLEMENTARY MATERIAL}

The Supplementary Material for this article can be found online at: https://www.frontiersin.org/articles/10.3389/fneur. 2021.647811/full\#supplementary-material 


\section{REFERENCES}

1. Wingerchuk DM, Carter JL. Multiple sclerosis: current and emerging diseasemodifying therapies and treatment strategies. Mayo Clin Proc. (2014) 89:22540. doi: 10.1016/j.mayocp.2013.11.002

2. Costello K, Kennedy P, Scanzillo J. Recognizing nonadherence in patients with multiple sclerosis and maintaining treatment adherence in the long term. Medscape J Med. (2008) 10:225. Available online at: https://pubmed.ncbi.nlm. nih.gov/19008986/

3. Evans C, Marrie RA, Zhu F, Leung S, Lu X, Melesse DY, Tremlett $\mathrm{H}$. Adherence and persistence to drug therapies for multiple sclerosis: a population-based study. Mult Scler Relat Disord. (2016) 8:78-85. doi: 10.1016/j.msard.2016.05.006

4. Setayeshgar S, Kingwell E, Zhu F, Zhang T, Carruthers R, Marrie $\mathrm{RA}$, et al. Persistence and adherence to the new oral diseasemodifying therapies for multiple sclerosis: a population-based study. Mult Scler Relat Disord. (2019) 27:364-9. doi: 10.1016/j.msard.2018. 11.004

5. Gajofatto A, Benedetti MD. Treatment strategies for multiple sclerosis: when to start, when to change, when to stop? World J Clin Cases. (2015) 3:545. doi: 10.12998/wjcc.v3.i7.545

6. Fagius J, Feresiadou A, Larsson EM, Burman J. Discontinuation of disease modifying treatments in middle aged multiple sclerosis patients. First line drugs vs. natalizumab. Mult Scler Relat Disord. (2017) 12:82-7. doi: 10.1016/j.msard.2017.01.009

7. Berger BA, Hudmon KS, Liang H. Predicting treatment discontinuation among patients with multiple sclerosis: application of the transtheoretical model of change. J Am Pharm Assoc. (2004) 44:445-54. doi: $10.1331 / 1544345041475607$

8. Meyniel C, Spelman T, Jokubaitis VG, Trojano M, Izquierdo G, Grand'Maison F. Country, sex, EDSS change and therapy choice independently predict treatment discontinuation in multiple sclerosis and clinically isolated syndrome. PLoS ONE. (2012) 7:e38661. doi: 10.1371/journal.pone. 0038661

9. Río J, Porcel J, Téllez N, Sánchez-Betancourt A, Tintoré MA, Arévalo $\mathrm{MJ}$, et al. Factors related with treatment adherence to interferon $\mathrm{b}$ and glatiramer acetate therapy in multiple sclerosis. Mult Scler J. (2005) 11:306-9. doi: 10.1191/1352458505ms1173oa

10. Tintoré $M$, Alexander $M$, Costello $K$, Duddy $M$, Jones DE, Law $\mathrm{N}$, et al. The state of multiple sclerosis: current insight into the patient/health care provider relationship, treatment challenges, and satisfaction. Patient Preference Adherence. (2017) 11:33. doi: 10.2147/PPA. S115090

11. O’Rourke KE, Hutchinson M. Stopping beta-interferon therapy in multiple sclerosis: an analysis of stopping patterns. Mult Scler J. (2005) 11:46-50. doi: 10.1191/1352458505ms1131oa

12. Kobelt G, Thompson A, Berg J, Gannedahl M, Eriksson J, MSCOI Study Group, et al. New insights into the burden and costs of multiple sclerosis in Europe. Mult Scler J. (2017) 23:1123-36. doi: 10.1177/13524585176 94432

13. Trojano $M$, Tintore $M$, Montalban $X$, Hillert J, Kalincik T, Iaffaldano $\mathrm{P}$, et al. Treatment decisions in multiple sclerosis-insights from real-world observational studies. Nat Rev Neurol. (2017) 13:105. doi: 10.1038/nrneurol.2016.188

14. Kalincik T, Butzkueven $H$. Observational data: understanding the real MS world. Mult Scler J. (2016) 22:1642-8. doi: 10.1177/1352458516 653667

15. Fox RJ, Salter AR, Tyry T, Sun J, You X, Laforet G, et al. Treatment discontinuation and disease progression with injectable disease-modifying therapies: findings from the north american research committee on multiple sclerosis database. Int J MS Care. (2013) 15:194-201. doi: 10.7224/1537-2073.2012-034

16. Fox RJ, Mehta R, Pham T, Park J, Wilson K, Bonafede M. (DXT62) Real-world treatment patterns in patients with multiple sclerosis using disease-modifying therapies. Int $J$ MS Care. (2020) 22:38.
Available online at: https://web.b.ebscohost.com/abstract?direct=true\& profile $=$ ehost\&scope $=$ site\&authtype $=$ crawler $\&$ jrnl $=15372073 \& \mathrm{AN}=$ 144209821\&h=f0Jp5fTwQG3jgUsrCWfeWV1lSxcMUeATtpC\%2bSJdi\%2b \%2fMFJmQSdLAzVBbH5WE6AEpyCr829jarImw1HkTpb1rBFQ\%3d\%3d\& $\mathrm{crl}=\mathrm{c} \&$ resultNs $=$ AdminWebAuth\&resultLocal=ErrCrlNotAuth\&crlhashurl= login.aspx\%3fdirect\%3dtrue\%26profile\%3dehost\%26scope\%3dsite \%26authtype\%3dcrawler\%26jrnl\%3d15372073\%26AN\%3d144209821

17. Turčáni $\mathrm{P}$, Mašková J, Húska J. Real-world treatment patterns of disease modifying therapy (DMT) for patients with relapse-remitting multiple sclerosis and patient satisfaction with therapy: results of the noninterventional SKARLET study in Slovakia. Patient Preference Adherence. (2020) 14:1129. doi: 10.2147/PPA.S254427

18. Daugherty KK, Butler JS, Mattingly M, Ryan M. Factors leading patients to discontinue multiple sclerosis therapies. J Am Pharm Assoc. (2005) 45:371-5. doi: 10.1331/1544345054003804

19. Jokubaitis VG, Spelman T, Lechner-Scott J, Barnett M, Shaw C, Vucic S, et al. The Australian Multiple Sclerosis (MS) immunotherapy study: a prospective, multicentre study of drug utilisation using the MSBase platform. PLoS ONE. (2013) 8:e59694. doi: 10.1371/journal.pone.0059694

Conflict of Interest: TS received compensation for serving on scientific advisory boards, honoraria for consultancy and funding for travel from Biogen; speaker honoraria from Novartis. MM has served on scientific advisory board for Biogen Idec and Teva and has received honoraria for lecturing from Biogen Idec, Merck Serono, Sanofi-Aventis and Teva. MM has received support for congress participation from Biogen Idec, Merck Serono, Novartis and Genzyme. PS has served on scientific advisory boards for Merck Serono, Teva, Novartis, Sanofi-Aventis and Biogen Idec and has received research support from Biogen Idec, Novartis and Sanofi-Aventis and received speaker honoraria from Merck Serono, Novartis, Teva, Sanofi-Aventis, Biogen Idec and Genzyme. HB received compensation for serving on scientific advisory boards and as a consultant for Biogen, Novartis; speaker honoraria from Biogen Australia, Merck Serono Australia, Novartis Australia; travel support from Biogen Australia, Merck Serono Australia; research support from the CASS Foundation (Australia), Merck Serono Australia, the Royal Melbourne Hospital. SV received consulting and lecturing fees, travel grants and research support from Biogen, Celgene, Genentech, Genzyme, Medday pharmaceuticals, Merck Serono, Novartis, Roche, Sanofi Aventis, and Teva Pharma. MT has served on scientific Advisory Boards for Biogen, Novartis, Roche, and Genzyme; has received speaker honoraria and travel support from Biogen Idec, Sanofi-Aventis, Merck Serono, Teva, Genzyme and Novartis; and has received research grants for her Institution from Biogen Idec, Merck Serono, and Novartis. PI has served on scientific advisory boards for Biogen Idec, Bayer, Teva, Roche, Merck Serono, Novartis, and Genzyme and has received funding for travel and/or Speaker honoraria from Sanofi Aventis, Genzyme, Biogen Idec, Teva, Merck Serono, and Novartis. FP is an employee of Biogen. RH is an employee of Biogen and holds stock. JH has received honoraria for serving on advisory boards for Biogen, Sanofi-Genzyme, and Novartis and speaker's fees from Biogen, Novartis, Merck-Serono, Bayer-Schering, Teva, and Sanofi-Genzyme. $\mathrm{JH}$ has served as PI for projects, or received unrestricted research support from BiogenIdec, Merck-Serono, TEVA, Sanofi-Genzyme, and Bayer-Schering. JH MS research is funded by the Swedish Research Council and the Swedish Brain Foundation.

The remaining authors declare that the research was conducted in the absence of any commercial or financial relationships that could be construed as a potential conflict of interest.

Copyright @ 2021 Hillert, Magyari, Soelberg Sørensen, Butzkueven, Van Der Welt, Vukusic, Trojano, Iaffaldano, Pellegrini, Hyde, Stawiarz, Manouchehrinia and Spelman. This is an open-access article distributed under the terms of the Creative Commons Attribution License (CC BY). The use, distribution or reproduction in other forums is permitted, provided the original author(s) and the copyright owner(s) are credited and that the original publication in this journal is cited, in accordance with accepted academic practice. No use, distribution or reproduction is permitted which does not comply with these terms. 